hep-th/0408124

SNUST-040801

\title{
On Solvable Time-Dependent Model and Rolling Closed String Tachyon
}

\author{
Yasuaki Hikida1 1 and Tadashi Takayanagi 2 \\ ${ }^{1}$ School of Physics $\&$ BK-21 Physics Division, Seoul National University \\ Seoul 151-747, Korea \\ ${ }^{2}$ Jefferson Physical Laboratory, Harvard University \\ Cambridge, MA 02138, USA
}

\begin{abstract}
We investigate the $S L(2, \mathbb{R}) / U(1)$ WZW model with level $0<k<2$ as a solvable time-dependent background in string theory. This model is expected to be dual to the one describing a rolling closed string tachyon with a time-like linear dilaton. We examine its exact metric and minisuperspace wave functions. Two point functions and the oneloop vacuum amplitude are computed and their relation to the closed string emission is discussed. Comparing with the results from the minisuperspace approximation, we find a physical interpretation of our choice to continue the Euclidean model into the Lorentzian one. Three point functions are also examined.
\end{abstract}

\footnotetext{
1 E-mail: hikida@phya.snu.ac.kr

2 E-mail: takayana@bose.harvard.edu
} 


\section{Introduction}

The open string tachyon condensation has been widely investigated up to now, and we obtain much insights on the decay process of unstable D-branes. However, it is very difficult to analyze closed string tachyon condensation except for the successful examples of the localized closed string tachyons initiated in [1] on non-supersymmetric orbifolds]. This is because of the lack of tractable conformal field theories for closed string tachyon condensation, which would change the background itself. Nevertheless, there have been some attempts to describe the homogeneous rolling tachyon condensation of closed strings by using a Lorentzian continuation of the bulk Liouville theory [3] [4] [5] (we call this the time-like Liouville theory). This idea is inspired by a similar process of open string tachyon condensation [6] and the related time-like boundary Liouville theory [7] [8] [9]. The closed string tachyon condensation in two dimensional string theory was recently discussed in [10] [11] by applying the matrix model description.

It is also very interesting to study inhomogeneous closed string tachyon condensations such as a tachyon kink, just like the open string case. The simplest example may be obtained from a Lorentzian continuation of sine-Liouville theoryt. The usual sine-Liouville theory is defined by the action (we always assume $\alpha^{\prime}=1$ below)

$$
S=\int d z^{2}\left[\partial X \bar{\partial} X+\partial \phi \bar{\partial} \phi+\lambda e^{b \phi} \cos \left(\frac{X}{R}\right)\right]
$$

where $\phi$ is a Liouville field with background charge $Q$ (such that the string coupling $\left.g_{s}=e^{-Q \phi}\right)$, and $X$ is a free boson compactified on a circle with radius $R$. The total central charge is $c=2+6 Q^{2}$ and the conformal dimension of the primary field $e^{b \phi}$ is $\Delta_{b}=-\frac{1}{4} b(b+2 Q)$.

Performing the Lorentzian continuations $\phi=-i X^{0}$ and $b=i \beta\left(X^{0}\right.$ and $\beta$ are real), we obtain

$$
S=\int d z^{2}\left[\partial X \bar{\partial} X-\partial X^{0} \bar{\partial} X^{0}+\lambda e^{\beta X^{0}} \cos \left(\frac{X}{R}\right)\right]
$$

1 For recent developments on this subject see reviews [2] and references therein.

2 One can find in 12 earlier discussions on the condensation of a tachyon kink using the Euclidean sine-Liouville theory or equally a non-critical string with a tachyon kink. 
Obviously, this action describes the condensation of a tachyon kink on the circle. Even before the Lorentzian continuation, sine-Liouville theory may not be easily solved exactly for generic parameters. However, at the specific value of parameters

$$
Q=\frac{1}{\sqrt{k-2}}, \quad R=\frac{1}{\sqrt{k}}, \quad b=-\frac{1}{Q}=-\sqrt{k-2},
$$

we can solve it by making use of the Fateev-Zamolodchikov-Zamolodchikov (FZZ) duality (see e.g. [13] 14] 15] 16]), which relates the theory to the $S L(2, \mathbb{R}) / U(1)$ WZW model with level $k$ (and radius $\tilde{R}=\frac{1}{R}=\sqrt{k}$ ). This is a sort of T-duality and can be proved explicitly in the case of $\mathcal{N}=2$ Liouville theory [17]. If we extend this duality relation to the region $0<k<2$ analytically, then we can analyze the previous inhomogeneous closed string tachyon condensation (1.2) using the relation to the $S L(2, \mathbb{R}) / U(1)$ WZW model. The latter model is much more useful because of not only technical reasons (e.g., of computing correlation functions) but also intuitive understandings. Indeed we can find an exact metric in the dual picture as we will see later.

Motivated by these facts, in this paper we mainly study the $S L(2, \mathbb{R}) / U(1)$ theory extended to the region $0<k<2$, where $R$ is real and $Q$ and $b$ are pure imaginary. As in the sine-Liouville theory, all of the physical parameters become real by regarding the linear dilaton field (in a free field realization) as a time coordinate. This coset model itself, of course, gives also an interesting solvable time-dependent background in string theory.

The rest of the paper is organized as follows. In section 2 we examine the geometry of the time-dependent background using the exact metric with stringy corrections. In section 3 we apply the minisuperspace approximation to our model and examine the wave functions. In section 4 we compute the exact two point function and discuss its relation to the closed string pair creation. We also find the exact three point function. In section 5 we analyze the decay of the vacuum by using the one-loop torus partition function. In section 6 we comments on the relation to the rolling closed string tachyon, and in section 7 we summarize the conclusions and discuss future problems.

3 See [18] 19]20] 20] for earlier discussions on cosmological interpretations of $S L(2, \mathbb{R}) / U(1)$ model. Also refer to e.g. a review [23] for recent developments of time-dependent backgrounds in string theory. 


\section{Exact Time-Dependent Geometry}

Originally, the $S L(2, \mathbb{R}) / U(1)$ WZW model $\mathbb{\theta}^{-}$was investigated to describe the Euclidean two dimensional black hole in the context of string theory [24] 225]. The level of the WZW model is labeled by $k$, and the exact geometry of the model is known including $\alpha^{\prime}$ correction [26] [27]. Roughly speaking, the quantum correction shifts the level $k \rightarrow k-2$, and the Euclidean signature of the metric requires $k>2$. If we go beyond this bound into $0<k<2$, then we obtain an interesting Lorentzian model as we will see below.

\subsection{Exact Geometry of $S L(2, \mathbb{R}) / U(1)$ Model with $0<k<2$}

In order to see the geometry given by the $S L(2, \mathbb{R}) / U(1)$ model, we should use the metric with $\alpha^{\prime}$ corrections since the shift -2 of the level $k$ is relevant in the action (1.2) and the parameters (1.3). Fortunately, the $\alpha^{\prime}$ exact metric of the model can be found in [26] ${ }^{2}$ as (we always assume $\alpha^{\prime}=1$ below)

$$
d s^{2}=(k-2)\left(d r^{2}+\frac{\tanh ^{2} r}{1-\frac{2}{k} \tanh ^{2} r} d \theta^{2}\right),
$$

where $\theta$ is compactified as $\theta \sim \theta+2 \pi$ and $r$ is restricted to $r \geq 0$. The string coupling is given by

$$
g_{s}^{2}=e^{2 \Phi}=\frac{1}{\cosh r \sqrt{\cosh ^{2} r-\frac{2}{k} \sinh ^{2} r}} .
$$

This metric represents a cigar-like geometry with the asymptotic radius $\tilde{R}=\sqrt{k}$, and, in particular for $k=9 / 4$, the critical string model describes the two dimensional (Euclidean)

4 There are two types of $U(1)$ symmetry in the $S L(2, \mathbb{R})$ WZW model; one is time-like and the other is space-like. We obtain the Euclidean (Lorentzian) geometry by gauging time-like (space-like) $U(1)$, which corresponds to the Euclidean (Lorentzian) 2d black hole. In this paper we mean the $S L(2, \mathbb{R}) / U(1)$ model as the one gauged by time-like $U(1)$.

5 The Lorentzian $S L(2, \mathbb{R}) / U(1)$ model with negative level $k<0$ also leads to a different Lorentzian geometry, which was investigated in [22]. We will not mainly discuss the case since we are motivated by the dual background with the inhomogeneous closed string tachyon condensation as discussed in section 1 . See also section 7 on this point.

6 For a general $\alpha^{\prime}$ the metric $d s^{2}$ is modified by overall factor $\alpha^{\prime}$. If we take the large $k$ limit, then the metric reduces to the familiar two dimensional black hole metric $d s^{2}=k \alpha^{\prime}\left(d r^{2}+\right.$ $\left.\tanh ^{2} r d \theta^{2}\right)$ with $g_{s}^{2}=1 / \cosh ^{2} r$. 
black hole [24] 25]. The exactness of the metric was proven in [27] by noticing the quantum corrections of the levels of the coset WZW model (see also 28] 29] for further discussions).

In order to define a consistent critical string theory, we have to add other conformal field theories with total central charge $c=24-6 /(k-2)$. We can assume, for example, that the additional sector consists of 24 scalar bosons $X_{2}, X_{3}, \cdots, X_{25}$ with a non-trivial linear dilaton. There are many other candidates for such an additional sector, but the sector does not play an important role in our discussions. Because the total system always preserves conformal symmetry, the backgrounds with any value of $k$ correspond to a series of exact solutions to the $\alpha^{\prime}$ corrected Einstein-dilaton gravity for bosonic string theory. Therefore, it must be possible to obtain an exact classical solution continuing into the region $0<k<2$.

Using the Lorentzian continuation of the level $k$, we obtain the following metric and dilaton日 (we also replace $r$ with $t$ to make its signature clear)

$$
\begin{aligned}
d s^{2} & =-\alpha d t^{2}+\alpha \frac{\tanh ^{2} t}{\frac{2}{2-\alpha} \tanh ^{2} t-1} d \theta^{2} \\
g_{s}^{2} & =\frac{1}{\cosh t \sqrt{\frac{2}{2-\alpha} \sinh ^{2} t-\cosh ^{2} t}}
\end{aligned}
$$

where we defined

$$
\alpha \equiv 2-k>0
$$

This metric and the dilaton have a space-like singularity at $t= \pm t^{*}$ (curvature is divergent), where $t^{*}>0$ is a solution to $\frac{2}{2-\alpha} \tanh ^{2} t-1=0$. Therefore, we use a region $t^{*}<t<\infty$ to define the spacetime, though we could use the other region $-\infty<t<-t^{*}$, which can be obtained by replacing $t \rightarrow-t$. The restriction of the coordinate is natural since the original metric is defined only for $r<0$ or $r>0$.

Let us see the asymptotic behaviors of the metric. At the late time $t \gg 1$ the background approaches to a flat spacetime (with the asymptotic radius $\tilde{R}=\sqrt{2-\alpha}$ ) with a time-like linear dilaton $g_{s} \sim e^{-t}$. Near the starting point $t^{*}$, where the metric and the dilaton diverge, we find the approximation of the metric and the dilaton

$$
d s^{2}=-\alpha d t^{2}+\left(\frac{2-\alpha}{2}\right)^{\frac{3}{2}} \frac{d \theta^{2}}{t-t^{*}}, \quad g_{s}^{2}=\sqrt{\frac{\alpha}{(4-2 \alpha)\left(t-t^{*}\right)}} .
$$

7 We use the fact that the dilaton value is determined only up to a constant shift. 
In conclusion, this time-dependent background describes a spacetime which starts with a singularity and contracts into a weakly coupled flat spacetime with a time-like linear dilaton.

So far we have discussed the geometry described by the string metric (2.3). It is also useful to examine other metrics such as the Einstein metric or the one observed by a D0-brane. Each of them is defined by $\left(d s^{\prime}\right)^{2}=\left(g_{s}\right)^{a} d s^{2}$, where the constant $a$ is $-1 / 6$ for the Einstein metric and -2 for the D0-brane metric. It is easy to see that the both metrics again have the similar singularity at $t= \pm t^{*}$.

\subsection{Exact Geometry of T-dual Model}

In the $S L(2, \mathbb{R}) / U(1)$ model, we can find the metric after the T-duality along the compactified circle including $\alpha^{\prime}$ correction. For the Euclidean case $k>2$, the exact metric for the T-dual model is given in [26]

$$
\begin{aligned}
d s^{2} & =(k-2)\left(d r^{2}+\frac{d \tilde{\theta}^{2}}{\tanh ^{2} r-\frac{2}{k}}\right), \\
g_{s}^{2} & =\frac{1}{\sinh r \sqrt{\sinh ^{2} r-\frac{2}{k} \cosh ^{2} r}},
\end{aligned}
$$

where $\tilde{\theta}$ is the dual coordinate of $\theta$ and is compactified as $\tilde{\theta} \sim \tilde{\theta}+2 \pi / k$. As before we obtain the exact time-dependent background with $0<k<2$ using the Lorentzian continuation as

$$
\begin{aligned}
d s^{2} & =-\alpha d t^{2}+\frac{\alpha d \tilde{\theta}^{2}}{\frac{2}{2-\alpha}-\tanh ^{2} t}, \\
g_{s}^{2} & =\frac{1}{\sinh t \sqrt{\frac{2}{2-\alpha} \cosh ^{2} t-\sinh ^{2} t}} .
\end{aligned}
$$

Interestingly, the metric has no singularity in contrast to the previous metric. The radius of the compactified direction starts from $R_{0}=\sqrt{\frac{\alpha}{2(2-\alpha)}}$ at the beginning $t=0$, and it approaches to $R_{\infty}=\frac{1}{\sqrt{2-\alpha}}$ at the late time $t=\infty$. Since the string coupling diverges at $t=0$, we can restrict the time coordinate to $t>0$. This is roughly consistent with the structure of the coordinate system (2.3) before the T-duality.

8 We can use $t<0$ by replacing $t \rightarrow-t$. 
At the late time $t \gg 1$, the background approaches to a flat spacetime with a timelike linear dilaton $g_{s} \sim e^{-t}$. Naively, the absence of singularity at $t= \pm t^{*}$ does not seem consistent with the geometry before the T-dual transformation. Even though we do not have a complete answer to this question, it is probable that the singularity at $t= \pm t^{*}$ in the original metric is actually not true one in string theory. This observation may be deduced from the analysis of the minisuperspace approximation in the next section. The wave function does not have a singular behavior at $t= \pm t^{*}$ and we can indeed reproduce the exact two point function computed later by just assuming the boundary condition at $t=0$ neglecting 9 the 'singularity' at $t= \pm t^{*}$. Notice also that such a 'discrepancy' already exists in the Euclidean theory.

Although the string metric of this background is smooth everywhere, the singularity $t=0$ appears in the dilaton field. Therefore, the other kinds of metrics (Einstein metric and D0-brane metric), discussed in the end of the previous subsection, become singular at $t=0$. Rewriting such metrics in a form $d s^{2}=-d t^{2}+a(t)^{2} d \theta^{2}$, we obtain the behavior $a(t) \sim t^{b}$ near $t=0$, where the constant $b$ is given by $b=\frac{1}{25}$ for the Einstein metric and $b=\frac{1}{3}$ for the D0-brane metric. This implies that the two dimensional spacetime gives an expanding behavior near the singularity $t=0$ for any kinds of metric considered so far. This is T-dual to the previous contracting spacetime.

\section{Minisuperspace Limit of the Time-Dependent Model}

Before moving to the exact conformal field theory analysis, we deal with the minisuperspace limit of string theory on (2.3) or (2.7). Finding the eigenfunction of the minisuperspace Hamiltonian means solving a Klein-Gordon equation for a scalar field 10 . We first concentrate on the case with (2.7) because the rolling tachyon model (1.2) is FZZ dual (Tdual) to the $S L(2, \mathbb{R}) / U(1)$ model and hence directly equivalent to the T-dual model (2.7).

9 Note also that the singularity of the dilaton coincides with that of the metric. It is possible that the both are canceled with each other.

10 For the minisuperspace analysis in Euclidean $S L(2, \mathbb{R})$ or $S L(2, \mathbb{R}) / U(1)$ model see e.g. [26] 30] [31]. 
From the exact metric (2.7), we find that the Laplacian $\Delta=-\frac{e^{2 \Phi}}{\sqrt{-g}} \partial_{\mu}\left(e^{-2 \Phi} \sqrt{-g} g^{\mu \nu} \partial_{\nu}\right)$ is

$$
\begin{aligned}
\Delta & =\frac{1}{\alpha}\left(\partial_{t}^{2}+2 \operatorname{coth} 2 t \partial_{t}+\left(\tanh ^{2} t-\frac{2}{2-\alpha}\right) \partial_{\tilde{\theta}}^{2}\right) \\
& =\frac{1}{\alpha}\left(4 \partial_{y}\left(y(1+y) \partial_{y}\right)+\left(-\frac{1}{1+y}+1-\frac{2}{2-\alpha}\right) \partial_{\tilde{\theta}}^{2}\right)
\end{aligned}
$$

with $y=\sinh ^{2} t$. The eigenfunctions of the Laplacian are given by linear combinations of two independent solutions, the regular one (note that $k n^{\prime} \in k \mathbb{Z}$ due to the periodicity $\tilde{\theta} \sim \tilde{\theta}+2 \pi / k)$

$$
\Phi^{(1)}=e^{i k n^{\prime} \tilde{\theta}}(1+y)^{\frac{k n^{\prime}}{2}} F\left(-j+\frac{k n^{\prime}}{2}, 1+j+\frac{k n^{\prime}}{2}, 1 ;-y\right),
$$

and the logarithmic one

$$
\begin{aligned}
\Phi^{(2)} & =e^{i k n^{\prime} \tilde{\theta}}(1+y)^{\frac{k n^{\prime}}{2}}\left(F\left(-j+\frac{k n^{\prime}}{2}, 1+j+\frac{k n^{\prime}}{2}, 1 ;-y\right) \ln (-y)\right. \\
+ & \sum_{l=1}^{\infty}(-y)^{l} \frac{\left(-j+\frac{k n^{\prime}}{2}\right)_{l}\left(1+j+\frac{k n^{\prime}}{2}\right)_{l}}{(l !)^{2}}\left\{\psi\left(-j+\frac{k n^{\prime}}{2}+l\right)-\psi\left(-j+\frac{k n^{\prime}}{2}\right)\right. \\
& \left.\left.+\psi\left(1+j+\frac{k n^{\prime}}{2}+l\right)-\psi\left(1+j+\frac{k n^{\prime}}{2}\right)-2 \psi(1+l)+2 \psi(1)\right\}\right),
\end{aligned}
$$

where we have defined

$$
(a)_{l}=\frac{\Gamma(a+l)}{\Gamma(a)}, \quad \psi(x)=\frac{d}{d x} \ln \Gamma(x) .
$$

The eigenvalues are 11

$$
\Delta=\frac{4 j(j+1)}{\alpha}+\frac{n^{\prime 2}}{(2-\alpha)} .
$$

Here we assume the boundary condition that the wave function should be smooth at $t=0$. Then the first solution $\Phi^{(1)}$ is selected since the second solution $\Phi^{(2)}$ diverges at $t=0$. After normalizing as 12

$$
\Psi=\frac{\Gamma\left(1+j+\frac{k n^{\prime}}{2}\right) \Gamma\left(1+j-\frac{k n^{\prime}}{2}\right)}{\Gamma(2 j+1)} \Phi^{(1)},
$$

11 The label $j$ should be written in terms of closed string oscillators and momenta of other directions if a critical string theory is considered.

12 We can show the self-adjointness of the minisuperspace Hamiltonian with the eigenfunction by following [30]. See [32] 33] for the analogue of the time-like Liouville theory. Our results, which are also consistent with the exact CFT result, almost corresponds to the specific case $\nu_{0}=0$ in [32]. 
we find its behavior for large $y\left(\sim\left(e^{t} / 2\right)^{2}\right)$

$$
\Psi \sim e^{i k n^{\prime} \tilde{\theta}} y^{j}+c_{n^{\prime}}^{j} e^{i k n^{\prime} \tilde{\theta}} y^{-1-j},
$$

with

$$
c_{n^{\prime}}^{j}=\frac{\Gamma\left(1+j+\frac{k n^{\prime}}{2}\right) \Gamma\left(1+j-\frac{k n^{\prime}}{2}\right) \Gamma(-2 j-1)}{\Gamma\left(-j+\frac{k n^{\prime}}{2}\right) \Gamma\left(-j-\frac{k n^{\prime}}{2}\right) \Gamma(2 j+1)} .
$$

Namely, for $j=-\frac{1}{2}+i \frac{\sqrt{\alpha}}{2} \omega(\omega \in \mathbb{R})$, we can interpret the eigenfunction as a linear combination of positive and negative frequency modes. The "reflection coefficient" (3.8) can be regarded as the two point function $\left\langle e^{i \omega X_{0}} e^{i \omega X_{0}}\right\rangle$ in our Lorentzian theory. This fact is well-known in the usual Liouville theory or the Euclidean $S L(2, \mathbb{R}) / U(1)$ model (see e.g. [34] 35] 36]). In the presence of the wall due to the screening operator, waves are reflected back into the weakly coupled region as $\psi(\phi) \sim e^{i p \phi}+S(p) e^{-i p \phi}$. Then it is obvious that the (non-trivial) two point function is given by $\left\langle e^{i p \phi} e^{i p \phi}\right\rangle=S(p)$. For our time-like case we extend this result by the Lorentzian continuation. As we will see in the next section, our result in the minisuperspace model is consistent with the one of the two point functions from the exact conformal field theory computations. Furthermore we later discuss that the reflection coefficient $c_{n^{\prime}}^{j}$ represents the closed string pair production as in the time-like Liouville theory [7] [8] [3] [4].

We can also apply the minisuperspace analysis to the original exact classical background (2.3). The Laplacian takes the similar form

$$
\begin{aligned}
\Delta & =\frac{1}{\alpha}\left(\partial_{t}^{2}+2 \operatorname{coth} 2 t \partial_{t}+\left(\operatorname{coth}^{2} t-\frac{2}{2-\alpha}\right) \partial_{\theta}^{2}\right) \\
& =\frac{1}{\alpha}\left(4 \partial_{y} y(1+y) \partial_{y}+\left(\frac{1}{y}+1-\frac{2}{2-\alpha}\right) \partial_{\theta}^{2}\right)
\end{aligned}
$$

with $y=\sinh ^{2} t$. There are two independent solutions which has the eigenvalue (3.5) (with $n^{\prime} \leftrightarrow n$ ) of the Laplacian (3.9) as in the previous case. Assuming the non-singular boundary condition at $t=0$ again, we get the solution $(n \in \mathbb{Z})$

$$
\Phi^{(1)}=e^{i n \theta} y^{\frac{|n|}{2}} F\left(-j+\frac{|n|}{2}, 1+j+\frac{|n|}{2}, 1+|n|,-y\right) .
$$

From this we can find the "reflection coefficient" (two point function)

$$
d_{n}^{j}=\frac{\left(\Gamma\left(1+j+\frac{|n|}{2}\right)\right)^{2} \Gamma(-2 j-1)}{\left(\Gamma\left(-j+\frac{|n|}{2}\right)\right)^{2} \Gamma(2 j+1)}
$$

and this is consistent with the exact results discussed later. Notice that in this argument the singularity of the metric and dilaton at $t= \pm t^{*}$ seems not important as mentioned in the previous section. 


\section{Exact Analysis of the Time-Dependent String Theory}

\subsection{Two Point Function}

We would like to turn to the exact conformal field theory analysis. For the Euclidean model of $S L(2, \mathbb{R}) / U(1)$ with $k>2$, the two point function was computed for the primaries $V_{j, m, \bar{m}}$, in 13

$\left\langle V_{j, m, \bar{m}} V_{j,-m,-\bar{m}}\right\rangle=(k-2)(\nu(k))^{2 j+1} \frac{\Gamma\left(1-\frac{2 j+1}{k-2}\right) \Gamma(-2 j-1) \Gamma(1+j-m) \Gamma(1+j+\bar{m})}{\Gamma\left(\frac{2 j+1}{k-2}\right) \Gamma(2 j+2) \Gamma(-j-m) \Gamma(-j+\bar{m})}$,

where

$$
\nu(k)=\frac{\Gamma\left(1+\frac{1}{k-2}\right)}{\pi \Gamma\left(1-\frac{1}{k-2}\right)} .
$$

When we shift the coupling constant, the function $\nu(k)$ scales as $\nu \sim\left(g_{s}\right)^{-2}$. The labels $(m, \bar{m})$ take the value

$$
m=\frac{n+k n^{\prime}}{2}, \quad \bar{m}=-\frac{n-k n^{\prime}}{2}, \quad n, n^{\prime} \in \mathbb{Z} .
$$

In the dual sine-Liouville model, the tachyon vertex operators $V_{j, m, \bar{m}}$ correspond to the operators

$$
V\left(p_{L}, p_{R}, \omega\right)=e^{-\frac{X^{0}}{\sqrt{\alpha}}} e^{i \omega X^{0}+i p_{L} X_{L}+i p_{R} X_{R}}
$$

where the relation between $(j, m, \bar{m})$ and $\left(\omega, p_{L}, p_{R}\right)$ is given by

$$
j=-\frac{1}{2}+i \frac{\sqrt{\alpha}}{2} \omega, \quad m=\frac{\sqrt{k}}{2} p_{L}, \quad \bar{m}=-\frac{\sqrt{k}}{2} p_{R} .
$$

Then the two point function for $0<k<2$ and $Q=\frac{i}{\sqrt{\alpha}}$ is given by

$$
\begin{aligned}
& \left\langle V\left(p_{L}, p_{R}, \omega\right) V\left(-p_{L},-p_{R}, \omega\right)\right\rangle \\
& =-(\nu(k))^{i \sqrt{\alpha} \omega} \frac{\Gamma\left(i \frac{\omega}{\sqrt{\alpha}}\right) \Gamma(-i \sqrt{\alpha} \omega) \Gamma\left(\frac{1}{2}+i \frac{\sqrt{\alpha}}{2} \omega-\frac{\sqrt{k}}{2} p_{L}\right) \Gamma\left(\frac{1}{2}+i \frac{\sqrt{\alpha}}{2} \omega-\frac{\sqrt{k}}{2} p_{R}\right)}{\Gamma\left(-i \frac{\omega}{\sqrt{\alpha}}\right) \Gamma(i \sqrt{\alpha} \omega) \Gamma\left(\frac{1}{2}-i \frac{\sqrt{\alpha}}{2} \omega-\frac{\sqrt{k}}{2} p_{L}\right) \Gamma\left(\frac{1}{2}-i \frac{\sqrt{\alpha}}{2} \omega-\frac{\sqrt{k}}{2} p_{R}\right)},
\end{aligned}
$$

13 It might be useful to observe that the two point function is invariant under $(-m, \bar{m}) \leftrightarrow$ $(m,-\bar{m})$ when comparing to the minisuperspace results. 
where

$$
\nu(k)=\frac{\Gamma(1-1 / \alpha)}{\pi \Gamma(1+1 / \alpha)} .
$$

Applying the FZZ duality to the time-dependent case, this result should also give the two point function of the time-dependent sine-Liouville theory defined by (1.2). We just suppressed the dependence on the parameter $\lambda$, which scales as $\left(g_{s}\right)^{\alpha}$.

The poles in the two point function (4.6) can be understood as follows. The poles $\omega=-i n / \sqrt{\alpha}(n \in \mathbb{Z})$ of $\Gamma(-i \sqrt{\alpha} \omega)$ correspond to the insertion of the screening operator $\sim e^{-2 Q \phi}=e^{-2 X^{0} / \sqrt{\alpha}}$ to the correlators in the free field representation of $S L(2, \mathbb{R}) / U(1)$ model. On the other hand, the poles $\omega=i n \sqrt{\alpha}$ coming from $\Gamma(i \omega / \sqrt{\alpha})$ can be explained as the contributions from the screening operator 14 or equally the tachyon perturbation $T\left(x_{0}, x\right)=\lambda e^{\sqrt{\alpha} X^{0}} \cos (\sqrt{2-\alpha} X)$ in the action (1.2). All other poles are reproduced 15 from the minisuperspace approximation done in section 3. Indeed we find that the minisuperspace results (3.8) and (3.11) are exactly the same as (4.6) for non-zero winding and momenta except the first factor $\Gamma(i \omega / \sqrt{\alpha})$, respectively. Missing this factor is very natural because it is related to the dual sine-Liouville theory and this duality occurs only in string theory and not in a field theory limit.

An interesting limit would be $\alpha=2(k=0)$. This should lead to a purely time-like Liouville theory $T=\lambda e^{\sqrt{2} X^{0}}$ as the cosine part becomes trivial. If we compute the two point function in this limit (we can set $p_{L}=p_{R}=0$ ), we get

$$
\langle V(\omega) V(\omega)\rangle=-(2 \pi)^{-i \sqrt{2} \omega} \frac{\Gamma\left(\frac{1}{2}+\frac{i \omega}{\sqrt{2}}\right)}{\Gamma\left(\frac{1}{2}-\frac{i \omega}{\sqrt{2}}\right)} .
$$

Indeed this is the same as that computed in the time-like Liouville theory [4]

$$
\langle V(\omega) V(\omega)\rangle=-\left(\frac{\pi \lambda \gamma\left(-\frac{1}{2}\right)}{4}\right)^{-\sqrt{2} i \omega} \frac{\Gamma\left(\frac{1}{2}+\frac{i \omega}{\sqrt{2}}\right)}{\Gamma\left(\frac{1}{2}-\frac{i \omega}{\sqrt{2}}\right)},
$$

after choosing a specific $\lambda$. It might be also useful to note that in this limit the metric (2.7) becomes flat and the string coupling $g_{s}^{2}$ is proportional to $\frac{1}{\sinh (2 t)}$. This represents the backreaction to the dilaton field (and no backreaction to the metric) in the time-like Liouville theory or equally the homogeneous closed string tachyon condensation.

14 Notice that only even power of the screening operator contributes due to the cosine combination.

15 The part obtained from the minisuperspace analysis can be also reproduced from a group theoretical consideration as $\int d^{2} x x^{j+m} \bar{x}^{j+\bar{m}}|x-1|^{-4(1+j)}$. 


\subsection{Closed String Pair Production}

In general a non-zero two point function $\left\langle e^{-i \omega X_{0}} e^{-i \omega X_{0}}\right\rangle$ implies that a pair of closed string with energy $\omega$ can be produced in that background as discussed in detail in the context of the time-like (boundary) Liouville theory [7] [8] [3] [4]. Thus the nonzero two point function (4.6) shows that this interesting phenomenon occurs also in our case. Since the production rate for each string mode is expected to be proportional 16 to $\left|\left\langle e^{-i \omega X_{0}} e^{-i \omega X_{0}}\right\rangle\right|^{2}=1$, the total closed string production can be estimated by summing over the Hagedorn tower of massive states with the density of state $\rho(\omega)$

$$
\int d \omega \rho(\omega)\left|\left\langle e^{-i \omega X_{0}} e^{-i \omega X_{0}}\right\rangle\right|^{2} \sim \int d \omega e^{4 \pi \sqrt{1+\frac{1}{4 \alpha}} \omega} .
$$

This is exponentially divergent and shows that there will be a huge backreaction due to the pair creation. The similar divergence was also observed in the time-like bulk Liouville

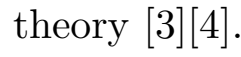

\subsection{Three Point Function}

We can also compute the three point function of the $S L(2, \mathbb{R}) / U(1)$ time-dependent model by extending the exact result [13] for $k>2$. It is given by

$$
\left\langle V_{j_{1}, m_{1}, \bar{m}_{1}} V_{j_{2}, m_{2}, \bar{m}_{2}} V_{j_{3}, m_{3}, \bar{m}_{3}}\right\rangle=D\left(j_{1}, j_{2}, j_{3}\right) \cdot F\left(j_{i}, m_{i}, \bar{m}_{i}\right) \cdot \delta^{2}\left(\sum_{i=1}^{3} m_{i}\right),
$$

where $F\left(j_{i}, m_{i}, \bar{m}_{i}\right)$ is a group theoretical coefficient

$$
\begin{aligned}
& F\left(j_{i}, m_{i}, \bar{m}_{i}\right)=\left(\prod_{i=1}^{3} \int d x_{i}^{2} x_{i}^{j_{i}+m_{i}} \bar{x}_{i}^{j_{i}+\bar{m}_{i}}\right) \\
& \cdot\left|x_{1}-x_{2}\right|^{2\left(j_{3}-j_{1}-j_{2}-1\right)}\left|x_{2}-x_{3}\right|^{2\left(j_{1}-j_{2}-j_{3}-1\right)}\left|x_{3}-x_{1}\right|^{2\left(j_{2}-j_{3}-j_{1}-1\right)},
\end{aligned}
$$

which is not possible to express in a simple way generically. For our time-dependent background $0<k<2$, we will substitute the values $j_{i}=-1 / 2+i \sqrt{\alpha} \omega_{i} / 2$ as before for

16 Here we have first taken the absolute valued square of the two point function and extends it to the Lorentzian region. Instead, if we first take the Lorentzian continuation, then we have either $\left|\left\langle e^{-i \omega X_{0}} e^{-i \omega X_{0}}\right\rangle\right|^{2}=1$ (e.g. for all cases in the region $0<k<1$ ) or $\left|\left\langle e^{-i \omega X_{0}} e^{-i \omega X_{0}}\right\rangle\right|^{2}=e^{-2 \pi \sqrt{\alpha \omega}}$ depending the value of the level $k$. Even in the latter case, it is overwhelmed by the exponentially large density of state and thus the closed string production is always exponentially divergent. 
the energy $\omega_{i}$ state. The non-trivial physics is included in the factor $D\left(j_{1}, j_{2}, j_{3}\right)$, which was computed in [35] 36] 37 as

$$
\begin{aligned}
& D\left(j_{1}, j_{2}, j_{3}\right)=\frac{k-2}{2 \pi^{3}} \nu(k)^{j_{1}+j_{2}+j_{3}+1} \\
& . \frac{G\left(-j_{1}-j_{2}-j_{3}-2\right) G\left(j_{3}-j_{1}-j_{2}-1\right) G\left(j_{2}-j_{1}-j_{3}-1\right) G\left(j_{1}-j_{2}-j_{3}-1\right)}{G(-1) G\left(-2 j_{1}-1\right) G\left(-2 j_{2}-1\right) G\left(-2 j_{3}-1\right)} .
\end{aligned}
$$

The function $G(j)$ is defined by

$$
G(j)=\tilde{b}^{-\tilde{b}^{2} j\left(j+1+\tilde{b}^{-2}\right)} \Upsilon^{-1}(-\tilde{b} j), \quad \tilde{b}=\frac{1}{\sqrt{k-2}}(=Q)
$$

in terms of the function $\Upsilon_{\tilde{b}}(x)$ introduced in [34. It might be convenient to express (4.13) as

$$
\begin{aligned}
& D\left(j_{1}, j_{2}, j_{3}\right)=\frac{1}{2 \pi^{3}} \nu(k)^{j_{1}+j_{2}+j_{3}+1} \tilde{b}^{-2 \tilde{b}^{2}\left(j_{1}+j_{2}+j_{3}+1\right)-1} \\
& \qquad \frac{\Upsilon(\tilde{b}) \Upsilon\left(\left(2 j_{1}+1\right) \tilde{b}\right) \Upsilon\left(\left(2 j_{2}+1\right) \tilde{b}\right) \Upsilon\left(\left(2 j_{3}+1\right) \tilde{b}\right)}{\Upsilon\left(\left(j_{1}+j_{2}+j_{3}+2\right) \tilde{b}\right) \Upsilon\left(\left(j_{1}+j_{2}-j_{3}+1\right) \tilde{b}\right) \Upsilon\left(\left(j_{3}+j_{1}-j_{2}+1\right) \tilde{b}\right) \Upsilon\left(\left(j_{2}+j_{3}-j_{1}+1\right) \tilde{b}\right)} .
\end{aligned}
$$

In order to obtain the three point function in our case, we perform the Lorentzian continuation to the region $0<k<2$.

Contrary to the two point function, it is not straightforward to continue to the region $0<k<2$ since the function $\Upsilon_{\tilde{b}}(x)$ is not originally defined for imaginary values of the parameter $\tilde{b}$ (see $(1.3))$. Fortunately we can accomplish the continuation procedure in a similar way as in [5] done for the time-like Liouville theory. The result is

$$
\begin{gathered}
D\left(\omega_{1}, \omega_{2}, \omega_{3}\right)=-\frac{1}{2 \pi^{3}}(\nu(k))^{-\frac{1}{2}+\frac{i \sqrt{\alpha}\left(\omega_{1}+\omega_{2}+\omega_{3}\right)}{2}} \cdot e^{-i \frac{\pi}{\alpha}} \cdot(\alpha)^{-\frac{i}{2 \sqrt{\alpha}}\left(\omega_{1}+\omega_{2}+\omega_{3}\right)+\frac{1}{2 \alpha}+\frac{3}{2}} \\
\cdot \frac{H\left(\frac{i}{\sqrt{\alpha}}\right) H\left(-2 \omega_{1}-\frac{i}{\sqrt{\alpha}}\right) H\left(-2 \omega_{2}-\frac{i}{\sqrt{\alpha}}\right) H\left(-2 \omega_{3}-\frac{i}{\sqrt{\alpha}}\right)}{H\left(-\omega_{1}-\omega_{2}-\omega_{3}\right) H\left(\omega_{3}-\omega_{1}-\omega_{2}\right) H\left(\omega_{2}-\omega_{1}-\omega_{3}\right) H\left(\omega_{1}-\omega_{2}-\omega_{3}\right)}
\end{gathered}
$$

where we define

$$
H(\omega)=\theta_{1}\left(-\frac{1}{2}+\frac{i \sqrt{\alpha} \omega}{2}, \alpha\right) \cdot Y_{\frac{1}{\sqrt{\alpha}}}(\omega)
$$

17 Notice that we are using a different convention of $j$ and it is related to that of [35] [36] via $j \rightarrow-j-1$. 
The function $Y_{\beta}(x)$, originally introduced 18 in [5], is written in the form

$$
\log Y_{\frac{1}{\sqrt{\alpha}}}(\omega)=\text { const. }+\int_{0}^{\infty} \frac{d \tau}{\tau}\left[e^{-\tau} \frac{(\omega+i \sqrt{\alpha})^{2}}{4}-\frac{\sin ^{2}\left(\frac{(\omega+i \sqrt{\alpha}) \tau}{4}\right)}{\sinh \left(\frac{\tau}{2 \sqrt{\alpha}}\right) \sinh \left(\frac{\sqrt{\alpha} \tau}{2}\right)}\right]
$$

Notice that the above formula becomes singular at $\alpha=1$ (or $k=1)$ due to the theta function in (4.17). The singularity can be analyzed in the same way as [5].

\section{Torus Amplitude and Decay Rate of the Vacuum}

Another quantity related to the closed string production may be (the imaginary part of) the one-loop vacuum amplitude 19. In the next subsections we compute the torus amplitude in the model with $S L(2, \mathbb{R}) / U(1) \times(D-2)$ free bosons. We find that the torus amplitude diverges, which may be rewritten as the imaginary part by an analytical continuation. We interpret the imaginary part as the decay rate of the vacuum (see e.g. [40] [41]). In the other subsections we see that there are two types of origin of the divergence. One is the emission of the tachyonic modes just like the ordinary bosonic string theory on flat spacetime. The other is due to the poles of the density of states, and we propose that the divergence is interpreted as the emission of the closed strings with high energy as discussed before.

\subsection{One-loop Amplitude in the Time-Dependent String Theory}

First let us assume that the time-dependent string theory consists of $S L(2, \mathbb{R}) / U(1)$ with level $k>2$ and $D-2(=24-6 /(k-2))$ free bosons 20 . Then, it is straightforward

18 In the notation of [5] this is written as $Y_{\frac{1}{\sqrt{\alpha}}}\left(-\frac{i}{\sqrt{\alpha}} j\right)$.

19 In the case of open string rolling tachyon, there is a definite relation that the imaginary part of the cylinder amplitude and the square root of the amplitude of closed string emission are the same [38] [39]. We expect a similar relation between the torus amplitude and the rate of the closed string production (or two point function), although the explicit relation is unclear. Indeed our result in section 5.3 implies there is such a relation between them.

20 We choose a different additional sector from the one in section 2 just for convenience. 
to write down the modular invariant vacuum amplitude computed from the path-integral formulation 42] (see also 43] 44])

$$
\begin{aligned}
& Z=2 \sqrt{k(k-2)} \int_{\mathcal{F}} \frac{d \tau d \bar{\tau}}{\tau_{2}} \int_{0}^{1} d s_{1} d s_{2} \\
& \sum_{w, m=-\infty}^{\infty} \sum_{i} q^{h_{i}} \bar{q}^{\bar{h}_{i}} e^{4 \pi \tau_{2}\left(1-\frac{1}{4(k-2)}\right)-\frac{k \pi}{\tau_{2}}\left|\left(s_{1}+w\right) \tau-\left(s_{2}+m\right)\right|^{2}+2 \pi \tau_{2} s_{1}^{2}} \\
& \frac{1}{\left|\sin \left(\pi\left(s_{1} \tau-s_{2}\right)\right)\right|^{2}} \prod_{r=1}^{\infty} \frac{\left|1-e^{2 \pi i r \tau}\right|^{4}}{\left|1-e^{2 \pi i r \tau-2 \pi i\left(s_{1} \tau-s_{2}\right)}\right|^{2}\left|1-e^{2 \pi i r \tau+2 \pi i\left(s_{1} \tau-s_{2}\right)}\right|^{2}}
\end{aligned}
$$

We denote $h_{i}, \bar{h}_{i}$ as the conformal dimensions of $D-2$ free bosons and $q=\exp (2 \pi i \tau)$ $\left(\tau=\tau_{1}+i \tau_{2}\right)$ as the moduli of the torus. We perform the integral of $\tau$ in the fundamental region $\mathcal{F}$.

For the value $0<k<2$ considered now, we should perform the Lorentzian continuation of the above amplitude21. As was done in the Euclidean case [42], we can perform the Poisson resummation with respect to $m$ and introduce an extra integration of the continuous valuable $s$ ('energy') in order to get the partition function in terms of the operator formalism. One important point in our Lorentzian case is that we should deform the contour of the integral of $s$ into the imaginary axis $(\pi / 2$ rotation) as follows

$$
\frac{1}{2 i} \sqrt{\frac{\alpha}{\tau_{2}}}=\int_{-i \infty}^{i \infty} d s e^{4 \pi \tau_{2} s^{2} / \alpha}
$$

To make this explicit we define $s=i \tilde{s}$. The partition function in the end can be reduced to 22

$$
\begin{aligned}
Z(q) & =\operatorname{Tr}_{\mathcal{H}} q^{L_{0}-\frac{c}{24}} \bar{q}^{\bar{L}_{0}-\frac{c}{24}} \\
& =i V_{D-2} \int \frac{d^{D-2} p}{(2 \pi)^{D-2}} \int_{-\infty}^{\infty} d \tilde{s} \sum_{m, \bar{m}, N, \bar{N}} \rho(\tilde{s})(q \bar{q})^{\frac{\tilde{s}^{2}}{\alpha}+\frac{(\vec{p})^{2}}{4}-\frac{D-2}{24}} q^{\frac{m^{2}}{(2-\alpha)}+N} \bar{q}^{\frac{\bar{m}^{2}}{(2-\alpha)}+\bar{N}},
\end{aligned}
$$

21 Notice that the coefficient includes the imaginary factor $\sqrt{k-2}=-i \sqrt{\alpha}$ after the continuation. This implies that the spacetime is truly Lorentzian just as the volume factor in the case of the flat spacetime becomes $i V_{26}$ after the Wick rotation.

22 In the Euclidean case the poles from the density of state are identified as the existence of the discrete states [42]. Even in our case there is contribution from the poles, but it leads to the imaginary part contrary to the Euclidean case. 
where $N, \bar{N}$ represent physical closed string oscillators and the volume of $D-2$ dimensional space is denoted as $V_{D-2}$. The density of state $\rho(\tilde{s})$ is defined by

$$
\rho(\tilde{s})=\frac{1}{2 \pi} 2 \ln L-\frac{1}{2 \pi} \frac{d}{2 d \tilde{s}} \ln \frac{\Gamma\left(\frac{1}{2}+\tilde{s}-m\right) \Gamma\left(\frac{1}{2}+\tilde{s}+\bar{m}\right)}{\Gamma\left(\frac{1}{2}-\tilde{s}-m\right) \Gamma\left(\frac{1}{2}-\tilde{s}+\bar{m}\right)},
$$

where we take $L \rightarrow \infty$ limit23. This makes the first term diverge, which reflects the infinite volume in the time direction. The second term appears due to the time-dependence of the background. We can see that the possible divergence arises from the IR region $\tau_{2} \rightarrow \infty$ (due to the tachyon) and the poles of $\rho(\tilde{s})$.

\subsection{IR Divergence due to Tachyonic Modes}

From the expression of $(5.3)$, we can see that there is a divergence from the IR region $\tau_{2} \rightarrow \infty$ due to the existence of the tachyonic modes $N=\bar{N}=0$. Following [40] we regularize the divergence by an analytical continuation. We first split the amplitude into the finite and divergent pieces as

$$
Z=\int_{\mathcal{F}} \frac{d \tau_{1} d \tau_{2}}{\tau_{2}} Z(q)=\int_{<R} \frac{d \tau_{1} d \tau_{2}}{\tau_{2}} Z(q)+\int_{-\frac{1}{2}}^{\frac{1}{2}} d \tau_{1} \int_{R}^{\infty} \frac{d \tau_{2}}{\tau_{2}} Z(q)
$$

with a cut off $R$. Utilizing a formula 40] 46]

$$
\operatorname{Im} \int_{R}^{\infty} \frac{d x}{x} x^{-n} e^{(a+i \epsilon) x}= \begin{cases}\pi a^{n} / n ! & \text { for } a \geq 0 \\ 0 & \text { for } a<0\end{cases}
$$

we find that the imaginary part comes from the second term as

$$
\operatorname{Im} \frac{Z}{i V_{D-2}}=\pi \sum_{m} \int \frac{d^{D-2} p}{(2 \pi)^{D-2}} \int_{0}^{\hat{s}} d s \rho(\tilde{s}) .
$$

We have used

$$
\hat{s}=\sqrt{\alpha\left(\frac{D-2}{24}-\frac{m^{2}}{(2-\alpha)}-\frac{\vec{p}^{2}}{4}\right)},
$$

and the level matching condition $L_{0}=\bar{L}_{0}$ coming from the $\tau_{1}$ integration. The inside of the square root must be positive. This imaginary part (5.7) implies the instability of the vacuum due to the existence of the tachyon, which was discussed in [40] for the flat spacetime and in [22] for the time-like linear dilaton. The novelty here is the modification of the density of the tachyon modes due to the time-dependence.

23 The similar formula can be also obtained for the usual Liouville theory (say, at the value $\alpha=2$ discussed later). In the case of $c \leq 1$ string theory (e.g. see a review [45]), the matrix model dual reproduces only the bulk term (the first term in (5.4)) since we can only see the quantities which depend on the cutoff $L$ due to the double scaling limit. 


\subsection{Emission of Closed Strings with High Energy}

Apart from the IR divergence discussed in the previous subsection, there is another type of divergence coming from the poles of the density of states (5.4). Since the structure of poles is complicated, we consider only a special case $\alpha=2$, where the gauged WZW model is equivalent to the time-like Liouville theory and a free boson. Practically we set $m=\bar{m}=0$ and add another momentum $p_{1}$. Then we obtain

$$
\operatorname{Im} \frac{Z}{i V_{D-2}}=2 \pi \sqrt{2-\alpha} \int_{\mathcal{F}} \frac{d \tau_{1} d \tau_{2}}{\tau_{2}} \int \frac{d^{D-1} p}{(2 \pi)^{D-1}} e^{-\pi \tau_{2} \vec{p}^{2}}|\eta(\tau)|^{-2(D-2)} \sum_{n=0}^{\infty} e^{-2 \pi \tau_{2}\left(n+\frac{1}{2}\right)^{2}},
$$

where we pick up the poles located at $\tilde{s}=-1 / 2-n$. We have used the contour of integral of $\tilde{s}$ through just below the poles for $\tilde{s}<0$, just above for $\tilde{s}>0$ and large $|\tilde{s}|$ in the upper half plain, as is naturally derived from the deformation of the counter (5.2). The quantized momentum $n$ in the time direction (see (5.9)) may suggest a periodicity along the imaginary time or a thermal property as is similar to the decaying D-branes [38].

What is the physical interpretation of this vacuum instability? This model has only closed strings as degrees of freedom, so the energy coming from the vacuum decay has to be carried out by closed strings. Let us see the UV behavior of the imaginary part. For the purpose, it is convenient to use the domain which is obtained by $S$-transformation $(\tau \rightarrow$ $-1 / \tau)$ from the fundamental domain $\mathcal{F}$. Employing the Poisson resummation formula

$$
\sum_{n=0}^{\infty} e^{-2 \pi \tau_{2}\left(n+\frac{1}{2}\right)^{2}}=\frac{1}{\sqrt{\tau_{2}}} \sum_{w=0}^{\infty} \rho_{w} e^{-\frac{\pi w^{2}}{2 \tau_{2}}}=\sum_{w=0}^{\infty} \rho_{w} \int_{-\infty}^{\infty} d p_{0} e^{-\pi \tau_{2} p_{0}^{2}+\sqrt{2} \pi i p_{0} w}
$$

with

$$
\rho_{w}= \begin{cases}\frac{1}{2 \sqrt{2}} & \text { for } w=0 \\ \frac{(-1)^{w}}{\sqrt{2}} & \text { otherwise }\end{cases}
$$

we can see that in the UV region $\tau_{2} \rightarrow 0$ the leading contribution comes from $w=0$ sector. Since the equation (5.9) with (5.10) for $w=0$ becomes the vacuum amplitude for the theory with $D$ free bosons and ghosts, the amplitude diverges due to the Hagedorn tower of closed string states $\rho(\tau) \sim e^{\frac{\pi}{6}(D-2) \frac{1}{\tau_{2}}}$ in the UV region. Roughly speaking, this behavior seems consistent with the relation (4.10) between the two point function $\left|\left\langle e^{-i \omega t} e^{-i \omega t}\right\rangle\right|=1$ and the closed string emission rate. 


\section{Possible Relation to Rolling Closed String Tachyon Condensation}

Up to now we have investigated on the one side of the FZZ duality, namely, the $S L(2, \mathbb{R}) / U(1)$ WZW model with $0<k<2$. The model itself, of course, deserves to be investigated as it describes an interesting time-dependent background. The other side of the FZZ duality is given by the sine-Liouville theory

$$
S=\int d z^{2}\left[\partial X \bar{\partial} X-\partial X^{0} \bar{\partial} X^{0}+\lambda e^{\beta X^{0}} \cos \left(\frac{X}{R}\right)\right]
$$

parameterized by

$$
Q=\frac{i}{\sqrt{\alpha}}, \quad R=\frac{1}{\sqrt{2-\alpha}}, \quad \beta=\sqrt{\alpha}
$$

This is the model of $1+1$ dimensional spacetime (plus the $D-2$ dimensional flat space) with the time-dependent closed string tachyon field and the linear dilaton

$$
T\left(x_{0}, x\right)=\lambda e^{\sqrt{\alpha} X^{0}} \cos (\sqrt{2-\alpha} X), \quad g_{s}=e^{-\frac{x^{0}}{\sqrt{\alpha}}}
$$

In other words, the model represents the system with the closed string tachyon condensing inhomogeneously as time evolves. Therefore, we have a new solvable model of the dynamical process of closed string tachyon condensation via this duality. The closed string pair creation driven by the closed string tachyon condensation (6.3) should be dual to that in the time-dependent background (2.7).

Because the FZZ duality is a kind of T-duality, we expect that the geometry driven by the tachyon condensation is that of the T-dual of the $S L(2, \mathbb{R}) / U(1)$ model $(2.7)$. The cosmological background starts from the strongly coupled region, then expands for a while, and finally approaches to a flat spacetime with a small coupling constant. In the FZZ dual model, one starts in the strongly coupled region with a small tachyon field, and ends up with a weak coupling constant but with a large tachyon field. Then one may wonder why the tachyon becomes so large in the late time, though the FZZ-dual metric (2.7) approaches to the flat spacetime. 
We can propose an answer 24 to this question by considering the choice of the vacuum. In such a time-dependent background the choice of the vacuum is very important in general. As is clear from the minisuperspace analysis, we have infinitely many kinds of vacua by assigning various boundary conditions for the propagating field. In our case, we chose a specific vacuum with the smooth boundary condition at $t=0$ in the $S L(2, \mathbb{R}) / U(1)$ model. We regarded this vacuum as the correct one because the choice of the vacuum leads to the exact two point functions (4.6). In other words, our simple Lorentzian continuation selects this vacuum among other many possible ones. In the sine-Liouville theory side, this corresponds to a specific choice of 'vacuum 25 at the singularity $(t=0)$, and the tachyon field might have a large value at the late time. Indeed, the two point function (4.6) has poles which can be explained only by the tachyon field (6.3) regarded as a screening operator. If we use the wave function $\Psi \equiv g_{s}^{-1} \Phi$ that is canonical in the (sine-)Liouville theory [48], then the boundary condition can be rephrased into the requirement $\Psi(t=0)=0$. This seems to suggest that the real singularity at $t=0$ can not be seen from fundamental strings, which might give an interesting resolution of the cosmological singularity in string theory.

We should also note that we are considering the region $0<k<2$ where the quantum effects are important. In fact, even in the Euclidean $S L(2, \mathbb{R}) / U(1)$ model, the quantum effects may change the classical picture. The wave function [48] of the sine-Liouville perturbation $\Phi \sim e^{(Q-1 / Q) \phi}$ becomes large in the weakly coupled region for small $k(>2)$, which is opposite to the classical case with large $k$. We believe that this fact is also closely related to the behavior of the tachyon field discussed just before.

24 A similar issue appears in the two dimensional string theory (or $c=1$ string) by regarding the $b \rightarrow 1 / b$ duality [34] in the ordinary Liouville theory as a sort of FZZ duality in our sineLiouville case. The time-dependence of the cosmological constant $\sim e^{2 x^{0}}$ is opposite to that of the dual one $\sim e^{-2 x^{0}}$. In the recent work [47] done after this paper, some evidences that there is actually no such paradox were obtained by using the $c=1$ matrix model. In that context the dual cosmological constant is not relevant for physical quantities.

25 At the singularity it is difficult to define the positive (or negative) frequency modes. We regarded the boundary condition as the definition of the vacuum. This vacuum can be formally

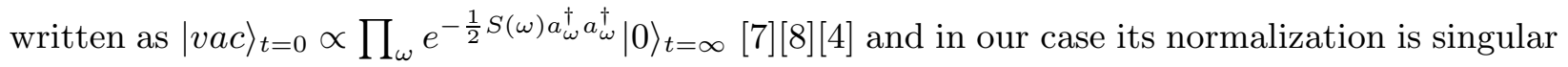
since the two point function $S(\omega)$ is a pure phase. 


\section{Conclusions and Discussions}

In this paper we investigated the $S L(2, \mathbb{R}) / U(1)$ WZW model with level $0<k<2$ as an exactly solvable time-dependent background in bosonic string theory. We examined the exact geometry of the model and analyzed the minisuperspace model. We read the two point functions from the difference of the two vacua (at the starting point and the far future) in the minisuperspace computations and found a match to the exact two point functions in the conformal field theory. In other words, the exact two point function given by continuing the Euclidean model $(k>2)$ to the Lorentzian one $(0<k<2)$ gives an important information on the choice of vacua. The non-zero two point function implies the closed string pair production, and we estimated the total production rate. We found that it diverges exponentially, which means that there is a very large backreaction at oneloop level. Indeed we showed that the imaginary part of its one-loop amplitude includes a similar divergence. We also calculated the exact three point function.

In addition to the advantage that various exact quantities can be obtained in the conformal field theory level, we have the exact metric including stringy corrections. This allows us to examine the cosmological structure of the two dimensional time-dependent spacetime. Furthermore, the model is expected to be T-dual (FZZ dual) to a background with an inhomogeneous and time-dependent closed string tachyon. Indeed we showed that the two point function in time-like bulk Liouville theory can be correctly reproduced as a particular case of our model. This duality suggests that the model with the rolling closed string tachyon is equivalent to the string theory on the cosmological background. This may lead to an interesting geometrical understanding of closed string tachyon condensation.

Another cosmological model can be defined by the Lorentzian $S L(2, \mathbb{R}) / U(1)$ WZW model with a negative level $k<0$. This model has also Lorentzian time-dependent geometry as a target space as investigated in [18] [19] 20][22] [21]. Applying the duality to the WZW model, we may obtain the model with the following closed string tachyon field

$$
T=e^{\sqrt{|k-2|} X^{0}} \cosh (\sqrt{|k|} X)
$$

The continuation of the original results for $k>2$ to this case $k<0$ seems to be highly non-trivial since the $X$ direction becomes decompactified in the negative level case. It would be interesting to investigate also this model furthermore. 


\section{Acknowledgments}

The authors are grateful to S. Minwalla, S. Mukohyama, S.-J. Rey, A. Strominger and H. Takayanagi for useful discussions. YH would like to thank the members of KEK for their hospitality and useful discussions. The work of TT was supported in part by DOE grant DE-FG02-91ER40654. 


\section{References}

[1] A. Adams, J. Polchinski and E. Silverstein, "Don't panic! Closed string tachyons in ALE space-times," JHEP 0110, 029 (2001) arXiv:hep-th/0108075.

[2] E. J. Martinec, "Defects, decay, and dissipated states," arXiv:hep-th/0210231; M. Headrick, S. Minwalla and T. Takayanagi, "Closed string tachyon condensation: An overview," Class. Quant. Grav. 21, S1539 (2004) arXiv:hep-th/0405064.

[3] B. C. Da Cunha and E. J. Martinec, "Closed string tachyon condensation and worldsheet inflation," Phys. Rev. D 68, 063502 (2003) arXiv:hep-th/0303087.

[4] A. Strominger and T. Takayanagi, "Correlators in timelike bulk Liouville theory," Adv. Theor. Math. Phys. 7, 369 (2003) [arXiv:hep-th/0303221].

[5] V. Schomerus, "Rolling tachyons from Liouville theory," JHEP 0311, 043 (2003) arXiv:hep-th/0306026.

[6] A. Sen, "Rolling tachyon," JHEP 0204, 048 (2002) arXiv:hep-th/0203211; "Tachyon matter," JHEP 0207, 065 (2002) arXiv:hep-th/0203265.

[7] A. Strominger, "Open string creation by S-branes," arXiv:hep-th/0209090.

[8] M. Gutperle and A. Strominger, "Timelike boundary Liouville theory," Phys. Rev. D 67, 126002 (2003) arXiv:hep-th/0301038.

[9] F. Larsen, A. Naqvi and S. Terashima, "Rolling tachyons and decaying branes," JHEP 0302, 039 (2003) arXiv:hep-th/0212248.

[10] J. L. Karczmarek and A. Strominger, "Matrix cosmology," JHEP 0404, 055 (2004) arXiv:hep-th/0309138.

[11] J. L. Karczmarek and A. Strominger, "Closed string tachyon condensation at $c=1$," JHEP 0405, 062 (2004) arXiv:hep-th/0403169; S. R. Das, J. L. Davis, F. Larsen and P. Mukhopadhyay, "Particle production in matrix cosmology," arXiv:hep-th/0403275; J. L. Karczmarek, A. Maloney and A. Strominger, "Hartle-Hawking vacuum for $\mathrm{c}=$ 1 tachyon condensation," arXiv:hep-th/0405092.

[12] D. Kutasov, "Irreversibility of the renormalization group flow in two-dimensional quantum gravity," Mod. Phys. Lett. A 7, 2943 (1992) arXiv:hep-th/9207064; E. Hsu and D. Kutasov, "The gravitational sine-Gordon model," Nucl. Phys. B 396, 693 (1993) arXiv:hep-th/9212023.

[13] A. Giveon and D. Kutasov, "Comments on double scaled little string theory," JHEP 0001, 023 (2000) arXiv:hep-th/9911039.

[14] V. Kazakov, I. K. Kostov and D. Kutasov, "A matrix model for the two-dimensional black hole," Nucl. Phys. B 622, 141 (2002) arXiv:hep-th/0101011.

[15] T. Fukuda and K. Hosomichi, "Three-point functions in sine-Liouville theory," JHEP 0109, 003 (2001) arXiv:hep-th/0105217.

[16] A. Giveon and D. Kutasov, "Notes on $A d S_{3}, "$ Nucl. Phys. B 621, 303 (2002) arXiv:hep-th/0106004. 
[17] K. Hori and A. Kapustin, "Duality of the fermionic 2d black hole and $N=2$ Liouville theory as mirror symmetry," JHEP 0108, 045 (2001) arXiv:hep-th/0104202.

[18] A. A. Tseytlin and C. Vafa, "Elements of string cosmology," Nucl. Phys. B 372, 443 (1992) arXiv:hep-th/9109048.

[19] C. Kounnas and D. Lust, "Cosmological string backgrounds from gauged WZW models," Phys. Lett. B 289, 56 (1992) arXiv:hep-th/9205046.

[20] D. Lust, "Cosmological string backgrounds," arXiv:hep-th/9303175.

[21] A. Buchel, P. Langfelder and J. Walcher, "On time-dependent backgrounds in supergravity and string theory," Phys. Rev. D 67, 024011 (2003) arXiv:hep-th/0207214.

[22] B. Craps, D. Kutasov and G. Rajesh, "String propagation in the presence of cosmological singularities," JHEP 0206, 053 (2002) arXiv:hep-th/0205101.

[23] L. Cornalba and M. S. Costa, "Time-dependent orbifolds and string cosmology," Fortsch. Phys. 52, 145 (2004) arXiv:hep-th/0310099].

[24] E. Witten, "On string theory and black holes," Phys. Rev. D 44, 314 (1991).

[25] G. Mandal, A. M. Sengupta and S. R. Wadia, "Classical solutions of two-dimensional string theory," Mod. Phys. Lett. A 6, 1685 (1991).

[26] R. Dijkgraaf, H. Verlinde and E. Verlinde, "String propagation in a black hole geometry," Nucl. Phys. B 371, 269 (1992).

[27] A. A. Tseytlin, "Effective action of gauged WZW model and exact string solutions," Nucl. Phys. B 399, 601 (1993) arXiv:hep-th/9301015.

[28] M. J. Perry and E. Teo, "Nonsingularity of the exact two-dimensional string black hole," Phys. Rev. Lett. 70, 2669 (1993) [arXiv:hep-th/9302037; P. Yi, "Nonsingular 2-D black holes and classical string backgrounds," Phys. Rev. D 48, 2777 (1993) arXiv:hep-th/9302070.

[29] T. Suyama and P. Yi, "A holographic view on matrix model of black hole," JHEP 0402, 017 (2004) arXiv:hep-th/0401078.

[30] J. Teschner, "The mini-superspace limit of the $S L(2, \mathbb{C}) / S U(2)$ WZNW model," Nucl. Phys. B 546, 369 (1999) arXiv:hep-th/9712258.

[31] S. Ribault and V. Schomerus, "Branes in the 2-D black hole," JHEP 0402, 019 (2004) arXiv:hep-th/0310024.

[32] S. Fredenhagen and V. Schomerus, "On minisuperspace models of S-branes," JHEP 0312, 003 (2003) arXiv:hep-th/0308205.

[33] Y. Hikida, "String theory on Lorentzian $A d S_{3}$ in minisuperspace," JHEP 0404, 025 (2004) arXiv:hep-th/0403081.

[34] A. B. Zamolodchikov and A. B. Zamolodchikov, "Structure constants and conformal bootstrap in Liouville field theory," Nucl. Phys. B 477, 577 (1996) arXiv:hepth/9506136].

[35] J. Teschner, "On structure constants and fusion rules in the $S L(2, \mathbb{C}) / S U(2)$ WZNW model," Nucl. Phys. B 546, 390 (1999) arXiv:hep-th/9712256. 
[36] J. Teschner, "Operator product expansion and factorization in the $\mathrm{H}_{3}^{+}$WZNW model," Nucl. Phys. B 571, 555 (2000) arXiv:hep-th/9906215].

[37] N. Ishibashi, K. Okuyama and Y. Satoh, "Path integral approach to string theory on $A d S_{3}$, , Nucl. Phys. B 588, 149 (2000) arXiv:hep-th/0005152.

[38] N. Lambert, H. Liu and J. Maldacena, "Closed strings from decaying D-branes," arXiv:hep-th/0303139.

[39] J. L. Karczmarek, H. Liu, J. Maldacena and A. Strominger, "UV finite brane decay," JHEP 0311, 042 (2003) arXiv:hep-th/0306132.

[40] N. Marcus, "Unitarity and regularized divergences in string amplitudes," Phys. Lett. B 219, 265 (1989).

[41] E. J. Weinberg and A. q. Wu, "Understanding complex perturbative effective potentials," Phys. Rev. D 36, 2474 (1987).

[42] A. Hanany, N. Prezas and J. Troost, "The partition function of the two-dimensional black hole conformal field theory," JHEP 0204, 014 (2002) arXiv:hep-th/0202129.

[43] K. Gawedzki, "Noncompact WZW conformal field theories," arXiv:hep-th/9110076.

[44] J. M. Maldacena, H. Ooguri and J. Son, "Strings in $A d S_{3}$ and the $S L(2, \mathbb{R})$ WZW model. II: Euclidean black hole," J. Math. Phys. 42, 2961 (2001) arXiv:hepth/0005183].

[45] I. R. Klebanov, "String theory in two-dimensions," arXiv:hep-th/9108019.

[46] B. Craps, P. Kraus and F. Larsen, "Loop corrected tachyon condensation," JHEP 0106, 062 (2001) arXiv:hep-th/0105227.

[47] T. Takayanagi, "Matrix model and time-like linear dilaton matter," arXiv:hepth/0411019.

[48] N. Seiberg, "Notes On Quantum Liouville Theory And Quantum Gravity," Prog. Theor. Phys. Suppl. 102, 319 (1990). 